\title{
LOW ENERGY EFFECTIVE LAGRANGIAN OF THE BESS MODEL
}

\author{
L. Anichini ${ }^{(a)}$, R. Casalbuoni ${ }^{(a, b)}$ and S. De $\operatorname{Curtis}^{(b)}$ \\ (a) Dipart. di Fisica, Univ. di Firenze, Largo E. Fermi, 2 - 50125 Firenze (Italy) \\ (b) I.N.F.N., Sezione di Firenze, Largo E. Fermi, 2 - 50125 Firenze (Italy)
}

\begin{abstract}
In this paper the low energy limit of the BESS model is studied in a systematic way. The method consists in eliminating the heavy vector field, by use of its classical equations of motion, in the infinite mass limit. After the elimination of the heavy degrees of freedom we get additional terms to the Standard Model lagrangian. After a finite renormalization of the ordinary gauge bosons wave functions, and redefinition of the lagrangian couplings in terms of $M_{Z}$, the fine structure constant and the Fermi constant, we can read directly the deviations from the Standard Model. By this procedure we can extend a result previously derived to the case in which the heavy vector bosons have a direct coupling to fermions. Consequences for the anomalous trilinear couplings are discussed.
\end{abstract}

Firenze Preprint - DFF-210/10/1994 


\section{Introduction}

The experimental data on electroweak interactions are giving more and more confirmations about the validity of the electroweak Standard Model (SM) up to energies of roughly 100 $\mathrm{GeV}$. One of the key ingredients of the SM is the electroweak symmetry breaking, for which, however, the theory does not provide an adequate mechanism. In fact, the minimal SM with a single Higgs field, given its well known patologies, can be considered, at most, a good parametrization of the symmetry breaking.

The solutions to this problem proposed so far can be divided into two broad classes depending if the Higgs is considered elementary or composite. Supersymmetric models belong to the first class, because supersymmetry gives rise naturally to the cancellation of the quadratic divergences in the Higgs self-mass, avoiding the fine tuning problem. To the second class belong all the models in which a new strong interaction theory is required at a scale of about $1 \mathrm{TeV}$. The prototype of these models is technicolor [1]. Whereas the models of the first class are weak interacting and therefore perturbatively calculable, in the other case life is more difficult. In fact, most of the calculations made in this area are based on the scaling from usual strong interactions. Other possibilities of dealing with the strong interacting sector, rely on the chiral perturbation theory. In fact the idea of technicolor is that the new strong interaction provides the dynamical breaking of $S U(2)_{L} \otimes U(1)_{Y}$. Then, one can use the chiral approach to write down an effective Lagrangian describing the Goldstone bosons arising from the symmetry breaking and which represent the longitudinal degrees of the $W$ and $Z$ mesons [2]. Typically this expansion is arrested to the fourth order and one extrapolates to higher energies using some unitarization procedures [5].

Another possibility is to introduce in the effective theory, in addition to the Goldstone bosons, other resonances due to the strong interaction. Such possibility was considered several years ago in the context of the BESS model (BESS stands for Breaking Electroweak Symmetry Breaking) [3], where, on the basis of chiral invariance, vector resonances are introduced. The phenomenology of this model has been studied in various papers as well as the limitations on its parameters arising from weak-interaction experiments. Regarding this last point, the studies have been done mainly numerically [4] or, in special case, analitically [6].

In this paper we want to study the problem of the experimental limitations on the BESS parameter space in a more general way. This treatment will give us the possibility to generalize it to the model which includes also axial-vector resonances, that have been already proposed in ref. [7].

The idea is very simple: we eliminate the fields of the vector resonances from the Lagrangian via their classical equation of motions in the limit of infinite mass, which in physical terms means that the mass must be much bigger than $M_{W}$. By defining in a convenient way this limit, we get an effective Lagrangian in terms of $W, Z$ and the photon fields, from which after a finite renormalization [8] we can read directly the deviations from the SM.

In Sect. 2 we review the basic ingredients of the BESS model. In Sect. 3 we perform the low-energy limit by considering $M_{V} \rightarrow \infty$ (limit that will be conveniently defined later on), $V$ being the new resonances. In Sect. 4 we identify the physical quantities by performing fields and couplings renormalization. The results are in Sect. 5 and 6 . 
The low-energy effects of the $V$ particles are discussed in Sect. 5 in terms of the $\epsilon$ parameters, and bounds on the BESS parameter space from the most recent experimental measurements are derived. In Sect. 6 we calculate the corrections to the trilinear gauge boson couplings which will be of great phenomenological interest for the studies at the future $e^{+} e^{-}$colliders. For completeness we give also the expressions for the anomalous quadrilinear couplings. Finally we discuss the results in Sect. 7.

\section{The BESS model}

An effective description of the symmetry breaking mechanism in electroweak theories can be done in terms of a non linear $\sigma$-model formulated on the quotient space of the breaking of $S U(2)_{L} \otimes S U(2)_{R} \rightarrow S U(2)_{L+R}$. This is the case when considering the limit of strong interacting Higgs sector $\left(M_{H} \rightarrow \infty\right)$.

As it is known [9], this non linear $\sigma$-model possesses a hidden local symmetry $H_{\text {local }}=$ $S U(2)_{V}$. Our assumption is that the appearing of this symmetry is realized through a new triplet of dynamical vector boson resonances $\mathbf{V}$ [3].

The main steps for the construction of the BESS model lagrangian are the following. One introduces the group variables $g(x) \in G=S U(2)_{L} \otimes S U(2)_{R}$

$$
g(x)=(L(x), R(x))
$$

with $L(x) \in S U(2)_{L}$ and $R(x) \in S U(2)_{R}$ which transform under $G \otimes H_{\text {local }}$ group as follows: $L \rightarrow g_{L} L h(x), R \rightarrow g_{R} R h(x)$ with $g_{L, R} \in S U(2)_{L, R}$ and $h(x) \in S U(2)_{V}$.

One further introduces the Maurer-Cartan form

$$
\omega_{\mu}=g^{\dagger} \partial_{\mu} g=\left(L^{\dagger} \partial_{\mu} L, R^{\dagger} \partial_{\mu} R\right)
$$

which can be decomposed into $\omega_{\mu}^{\|}$lying in the Lie algebra of $H_{\text {local }}$, and into the orthogonal complement $\omega_{\mu}^{\perp}$

$$
\begin{aligned}
\omega_{\mu}^{\|} & =\frac{1}{2}\left(L^{\dagger} \partial_{\mu} L+R^{\dagger} \partial_{\mu} R\right) \\
\omega_{\mu}^{\perp} & =\frac{1}{2}\left(L^{\dagger} \partial_{\mu} L-R^{\dagger} \partial_{\mu} R\right)
\end{aligned}
$$

Both $\omega_{\mu}^{\|}$and $\omega_{\mu}^{\perp}$ are singlet of $G$ and transform under $H_{\text {local }}$ as

$$
\begin{aligned}
\omega_{\mu}^{\|} & \rightarrow h^{\dagger} \omega_{\mu}^{\|} h+h^{\dagger} \partial_{\mu} h \\
\omega_{\mu}^{\perp} & \rightarrow h^{\dagger} \omega_{\mu}^{\perp} h
\end{aligned}
$$

The non linear $\sigma$-model Lagrangian describing the electroweak symmetry breaking sector can be easily reconstructed in terms of $\omega_{\mu}^{\perp}$

$$
\mathcal{L}=-v^{2} \operatorname{tr}\left(\omega_{\mu}^{\perp} \omega^{\perp \mu}\right)=\frac{v^{2}}{4} \operatorname{tr}\left(\partial_{\mu} U \partial^{\mu} U^{\dagger}\right)
$$

where $U=L R^{\dagger}$ is a singlet under $H_{\text {local }}$ and $v \simeq 250 \mathrm{GeV}$ is the standard electroweak scale. 
Introducing a triplet of gauge bosons $\mathbf{V}_{\mu}$ for the local group $S U(2)_{V}$, one can show that the most general Lagrangian, symmetric under $G \otimes H_{\text {local }}$ and under the parity transformation $L \leftrightarrow R$, containing at most two derivatives, can be constructed as an arbitrary combination of two invariant terms. Furthermore, assuming that the gauge bosons of the hidden symmetry become dynamical we get

$$
\begin{aligned}
\mathcal{L}= & -v^{2}\left[\operatorname{tr}\left(\omega_{\mu}^{\perp} \omega^{\perp \mu}\right)+\alpha \operatorname{tr}\left(\omega_{\mu}^{\|}-\mathbf{V}_{\mu}\right)^{2}\right] \\
& +\frac{2}{g^{\prime \prime 2}} \operatorname{tr}\left[F^{\mu \nu}(\mathbf{V}) F_{\mu \nu}(\mathbf{V})\right]
\end{aligned}
$$

with $\alpha$ an arbitrary parameter,

$$
F_{\mu \nu}(\mathbf{V})=\partial_{\mu} \mathbf{V}_{\nu}-\partial_{\nu} \mathbf{V}_{\mu}+\left[\mathbf{V}_{\mu}, \mathbf{V}_{\nu}\right]
$$

and $\mathbf{V}_{\mu}=\frac{i}{2} \frac{g^{\prime \prime}}{2} V_{\mu}^{a} \tau^{a}$, with $g^{\prime \prime}$ the new gauge coupling constant and $\tau^{a}$ the Pauli matrices.

The gauging of the standard $S U(2)_{L} \otimes U(1)_{Y}$ group is simply obtained by substituting in (2.3) the ordinary derivatives with covariant left and right derivatives acting on the left and right group elements respectively

$$
\begin{aligned}
\partial_{\mu} L & \rightarrow\left(\partial_{\mu}+\tilde{\mathbf{W}}_{\mu}\right) L \\
\partial_{\mu} R & \rightarrow\left(\partial_{\mu}+\tilde{\mathbf{Y}}_{\mu}\right) R
\end{aligned}
$$

where $\tilde{\mathbf{W}}_{\mu}=\frac{i}{2} \tilde{g} \tilde{W}_{\mu}^{a} \tau^{a}$ and $\tilde{\mathbf{Y}}_{\mu}=\frac{i}{2} \tilde{g}^{\prime} \tilde{Y}_{\mu} \tau^{3}$ and by adding the standard kinetic terms for $\tilde{\mathbf{W}}$ and $\tilde{\mathbf{Y}}$

$$
\mathcal{L}^{k i n}(\tilde{\mathbf{W}}, \tilde{\mathbf{Y}})=\frac{1}{2 \tilde{g}^{2}} \operatorname{tr}\left[F^{\mu \nu}(\tilde{\mathbf{W}}) F_{\mu \nu}(\tilde{\mathbf{W}})\right]+\frac{1}{2 \tilde{g}^{\prime 2}} \operatorname{tr}\left[F^{\mu \nu}(\tilde{\mathbf{Y}}) F_{\mu \nu}(\tilde{\mathbf{Y}})\right]
$$

with

$$
\begin{aligned}
F_{\mu \nu}(\tilde{\mathbf{W}}) & =\partial_{\mu} \tilde{\mathbf{W}}_{\nu}-\partial_{\nu} \tilde{\mathbf{W}}_{\mu}+\left[\tilde{\mathbf{W}}_{\mu}, \tilde{\mathbf{W}}_{\nu}\right] \\
F_{\mu \nu}(\tilde{\mathbf{Y}}) & =\partial_{\mu} \tilde{\mathbf{Y}}_{\nu}-\partial_{\nu} \tilde{\mathbf{Y}}_{\mu}
\end{aligned}
$$

Of course, if one assumes only the invariance under $S U(2)_{L} \otimes U(1)_{Y} \otimes S U(2)_{V}$ many other invariant terms are possible, but, for simplicity, we will ignore these extra terms in this paper.

Due to the gauge invariance of $\mathcal{L}$ we can choose the gauge with $L=R=1$ [3] (unitary gauge) and we get

$$
\mathcal{L}=-\frac{v^{2}}{4}\left[\operatorname{tr}\left(\tilde{\mathbf{W}}_{\mu}-\tilde{\mathbf{Y}}_{\mu}\right)^{2}+\alpha \operatorname{tr}\left(\tilde{\mathbf{W}}_{\mu}+\tilde{\mathbf{Y}}_{\mu}-2 \mathbf{V}_{\mu}\right)^{2}\right]+\mathcal{L}^{k i n}(\tilde{\mathbf{W}}, \tilde{\mathbf{Y}}, \mathbf{V})
$$

We have used tilded quantities to remember that, due to the effects of the $\mathbf{V}$ particles, they are not the physical parameters and fields. In the next sections we will derive the relations between the tilded quantities and the physical ones in the low-energy limit.

From eq. (2.11) one can easily derive the mass eigenstates and the mixing angles among the standard gauge bosons and the new resonances [3]. Furthermore, since in the 
limit $g^{\prime \prime} \rightarrow \infty$, the lagrangian $\mathcal{L}$ reproduces the SM terms, corrections to the SM relations come in powers of $1 / g^{\prime \prime}$.

Finally let us consider the fermions of the SM and denote them by $\psi_{L}$ and $\psi_{R}$. They couple to $\mathbf{V}$ via the mixing with the standard $\tilde{\mathbf{W}}$ and $\tilde{\mathbf{Y}}$. In addition, we also expect direct couplings to the new vector bosons since they are allowed by the symmetries of $\mathcal{L}[3]$. In fact, we can define Fermi fields transforming as doublets under the local group $S U(2)_{V}$ and singlets under the global one: $\chi_{L}=L^{\dagger} \psi_{L}$. We can then construct an invariant term acting on $\chi_{L}$ by the covariant derivative with respect to $S U(2)_{V}$. In the unitary gauge we get

$$
\begin{aligned}
\mathcal{L}_{\text {fermion }} & =\overline{\tilde{\psi}}_{L} i \gamma^{\mu}\left(\partial_{\mu}+\tilde{\mathbf{W}}_{\mu}+\frac{i}{2} \tilde{g}^{\prime}(B-L) \tilde{Y}_{\mu}\right) \tilde{\psi}_{L} \\
& +\overline{\tilde{\psi}}_{R} i \gamma^{\mu}\left(\partial_{\mu}+\tilde{\mathbf{Y}}_{\mu}+\frac{i}{2} \tilde{g}^{\prime}(B-L) \tilde{Y}_{\mu}\right) \tilde{\psi}_{R} \\
& +b \overline{\tilde{\psi}}_{L} i \gamma^{\mu}\left(\partial_{\mu}+\mathbf{V}_{\mu}+\frac{i}{2} \tilde{g}^{\prime}(B-L) \tilde{Y}_{\mu}\right) \tilde{\psi}_{L}
\end{aligned}
$$

where $B(L)$ is the baryon (lepton) number and $b$ is a new parameter. Notice that due to the introduction of the direct coupling of the $\mathbf{V}$ to the fermions, we have to rescale $\tilde{\psi}_{L}=(1+b)^{-1 / 2} \psi_{L}$ in order to get a canonical kinetic term for the fermions [3].

\section{The low-energy limit}

We want to study the effects of the $\mathbf{V}$ particles in the low-energy limit. This can be done by eliminating the $\mathbf{V}$ fields with the solution of their equations of motion for $M_{V} \rightarrow \infty$. In fact in this limit the kinetic term of the new resonances is negligible. Also, since their mass is given by (neglecting electromagnetic corrections) $M_{V}^{2} \simeq \alpha\left(v^{2} / 4\right) g^{\prime \prime 2}$, we will take the limit by fixing $g^{\prime \prime}$ and $v$ and sending $\alpha \rightarrow \infty$. Because $v$ is experimentally fixed, the only other possibility would be to send $g^{\prime \prime} \rightarrow \infty$, but, in this case, the $\mathbf{V}$ bosons would decouple.

We will study the effective theory by considering corrections up to order $\left(1 / g^{\prime \prime}\right)^{2}$.

Concerning the other parameter $b$, we expect it to be of the order $\left(1 / g^{\prime \prime}\right)^{2}$ as arising from $\mathbf{V}-\mathbf{W}$ mixing and one-loop corrections to the $\mathbf{W}$-fermion vertex and $\mathbf{W}$ propagator [3]. The present bounds from the LEP measurements, are consistent with this conjecture (see Sect. 5) so we will consider our effective theory as an expansion both in $1 / g^{\prime \prime}$ and $b$ in which terms of the order $b / g^{\prime \prime}$ are neglected.

Let us solve the equation of motion for $\mathbf{V}$ in this limit. By evaluating $\partial \mathcal{L} / \partial V_{\mu}^{a}$ from eqs. (2.11) and (2.12) we get

$$
\mathbf{V}_{\mu}=\frac{1}{2}\left(\tilde{\mathbf{W}}_{\mu}+\tilde{\mathbf{Y}}_{\mu}\right)
$$

By substituting this equation in the Lagrangian we get

$$
\begin{aligned}
\mathcal{L}_{\text {eff }} & =-\frac{v^{2}}{4} \operatorname{tr}\left(\tilde{\mathbf{W}}_{\mu}-\tilde{\mathbf{Y}}_{\mu}\right)^{2} \\
& +\frac{2}{g^{\prime \prime 2}} \operatorname{tr}\left[F^{\mu \nu}\left(\frac{\tilde{\mathbf{W}}+\tilde{\mathbf{Y}}}{2}\right) F_{\mu \nu}\left(\frac{\tilde{\mathbf{W}}+\tilde{\mathbf{Y}}}{2}\right)\right] \\
& +\mathcal{L}^{\text {kin }}(\tilde{\mathbf{W}}, \tilde{\mathbf{Y}})+\mathcal{L}_{\text {eff }}^{\text {charged }}+\mathcal{L}_{\text {eff }}^{\text {neutral }}+\bar{\psi} i \gamma^{\mu} \partial_{\mu} \psi
\end{aligned}
$$


where we have separated the charged and neutral fermionic sector

$$
\begin{aligned}
\mathcal{L}_{\text {eff }}^{\text {charged }} & =-\frac{\tilde{e}}{\sqrt{2} \tilde{s}_{\theta}}\left(1-\frac{b}{2}\right) \bar{\psi} \gamma^{\mu} \frac{1-\gamma_{5}}{2} \psi_{u} \tilde{W}_{\mu}^{-}+\text {h.c. } \\
\mathcal{L}_{\text {eff }}^{\text {neutral }} & =-\frac{\tilde{e}}{\tilde{s}_{\theta} \tilde{c}_{\theta}}\left(1-\frac{b}{2}\right) \bar{\psi} \gamma^{\mu}\left[T_{L}^{3} \frac{1-\gamma_{5}}{2}-Q \tilde{s}_{\theta}^{2}\left(1+\frac{b}{2}\right)\right] \psi \tilde{Z}_{\mu}-\tilde{e} \bar{\psi} \gamma^{\mu} Q \psi \tilde{A}_{\mu}
\end{aligned}
$$

and we have used the following standard definitions:

$$
\begin{aligned}
Q & =\frac{\tau^{3}}{2}+\frac{B-L}{2} \\
T_{L}^{3} \psi_{L} & =\frac{\tau^{3}}{2} \psi_{L} \quad T_{L}^{3} \psi_{R}=0 \\
\tilde{W}_{\mu}^{ \pm} & =\frac{1}{\sqrt{2}}\left(\tilde{W}_{1} \mp i \tilde{W}_{2}\right) \\
\tilde{W}_{\mu}^{3} & =\tilde{s}_{\theta} \tilde{A}_{\mu}+\tilde{c}_{\theta} \tilde{Z}_{\mu} \\
\tilde{Y}_{\mu} & =\tilde{c}_{\theta} \tilde{A}_{\mu}-\tilde{s}_{\theta} \tilde{Z}_{\mu} \\
\tilde{e} & =\tilde{g} \tilde{s}_{\theta}=\tilde{g}^{\prime} \tilde{c}_{\theta} \\
\psi & =\left(\begin{array}{c}
\psi_{u} \\
\psi_{d}
\end{array}\right)
\end{aligned}
$$

Notice that $\tilde{g}, \tilde{g}^{\prime}, \tilde{e}, \tilde{s}_{\theta}, \tilde{c}_{\theta}$ have the same definitions as in the SM. As stated before, due to the effects of the $\mathbf{V}$ particles, these are not the physical quantities in our model.

From eq. (3.2) we see that the effective contribution of the $\mathbf{V}$ particles give additional terms to the kinetic terms of the standard $\tilde{\mathbf{W}}$ and $\tilde{\mathbf{Y}}$. This will imply a renormalization of the fields in order to have canonical kinetic terms. By calling $\mathcal{L}_{\text {eff }}^{\text {kin }(2)}$ the bilinear terms coming from the kinetic terms for the gauge bosons, we have (using the definitions of eq. $(3.5))$

$$
\begin{aligned}
\mathcal{L}_{\text {eff }}^{\text {kin }(2)}\left(\tilde{W}^{ \pm}, \tilde{A}, \tilde{Z}\right) & =-\frac{1}{4}\left(1+z_{\gamma}\right) \tilde{A}_{\mu \nu} \tilde{A}^{\mu \nu}-\frac{1}{2}\left(1+z_{w}\right) \tilde{W}_{\mu \nu}^{+} \tilde{W}^{\mu \nu-} \\
& -\frac{1}{4}\left(1+z_{z}\right) \tilde{Z}_{\mu \nu} \tilde{Z}^{\mu \nu}+\frac{1}{2} z_{z \gamma} \tilde{A}_{\mu \nu} \tilde{Z}^{\mu \nu}
\end{aligned}
$$

where $O_{\mu \nu}=\partial_{\mu} O_{\nu}-\partial_{\nu} O_{\mu},\left(O=\tilde{W}^{ \pm}, \tilde{A}, \tilde{Z}\right)$ and

$$
z_{\gamma}=4 s_{\theta}^{2}\left(\frac{g}{g^{\prime \prime}}\right)^{2} \quad z_{w}=\left(\frac{g}{g^{\prime \prime}}\right)^{2} \quad z_{z}=\frac{c_{2 \theta}^{2}}{c_{\theta}^{2}}\left(\frac{g}{g^{\prime \prime}}\right)^{2} \quad z_{z \gamma}=-2 \frac{s_{\theta}}{c_{\theta}} c_{2 \theta}^{2}\left(\frac{g}{g^{\prime \prime}}\right)^{2}
$$

In eq. (3.7) we have not used the tilded quantities since these parameters are already of the order of $\left(1 / g^{\prime \prime}\right)^{2}$.

The corrections to $\mathcal{L}_{S M}$ are $U(1)_{e m}$ invariant and produce a wave-function renormalization of $\tilde{A}_{\mu}, \tilde{Z}_{\mu}, \tilde{W}_{\mu}^{ \pm}$plus a mixing term $\tilde{A}_{\mu}-\tilde{Z}_{\mu}$. Notice that in general there could be two other renormalization terms: $\delta M_{W}^{2} \tilde{W}_{\mu}^{+} \tilde{W}^{\mu-}$ and $\delta M_{Z}^{2} \tilde{Z}_{\mu} \tilde{Z}^{\mu}$ which, however, are zero in this model. In the next section we will absorb these corrections by a convenient redefinition of the fields. Actually there are only three renormalization transformations of the fields $\tilde{A}_{\mu}, \tilde{Z}_{\mu}, \tilde{W}_{\mu}^{ \pm}$without changing the physics. This means that three of the four deviations $z_{\gamma}, z_{w}, z_{z}, z_{z \gamma}$ are non physical, and this is consistent with the fact that they depend on a single parameter $g^{\prime \prime}$. 
Analogously we can calculate the corrections to the trilinear and quadrilinear terms coming from the kinetic terms of the gauge bosons. We get

$$
\begin{aligned}
\mathcal{L}_{e f f}^{k i n}{ }^{(3)}\left(\tilde{W}^{ \pm}, \tilde{A}, \tilde{Z}\right)= & i \tilde{g} \tilde{c}_{\theta}\left(1+z_{z w w}\right)\left[\tilde{Z}^{\mu \nu} \tilde{W}_{\mu}^{-} \tilde{W}_{\nu}^{+}+\tilde{Z}^{\nu}\left(\tilde{W}_{\mu \nu}^{-} \tilde{W}_{\mu}^{+}-\tilde{W}_{\mu \nu}^{+} \tilde{W}_{\mu}^{-}\right)\right] \\
& +i \tilde{e}\left(1+z_{w}\right)\left[\tilde{A}^{\mu \nu} \tilde{W}_{\mu}^{-} \tilde{W}_{\nu}^{+}+\tilde{A}^{\nu}\left(\tilde{W}_{\mu \nu}^{-} \tilde{W}_{\mu}^{+}-\tilde{W}_{\mu \nu}^{+} \tilde{W}_{\mu}^{-}\right)\right] \\
\mathcal{L}_{e f f}^{k i n}{ }^{(4)}\left(\tilde{W}^{ \pm}, \tilde{A}, \tilde{Z}\right)= & S_{\mu \rho \nu \sigma} \tilde{W}_{\mu}^{+} \tilde{W}_{\rho}^{-}\left[-\frac{\tilde{e}^{2}}{2}\left(1+z_{w}\right) \tilde{A}_{\nu} \tilde{A}_{\sigma}-\tilde{e} \tilde{g} \tilde{c}_{\theta}\left(1+z_{z w w}\right) \tilde{A}_{\nu} \tilde{Z}_{\sigma}\right. \\
& \left.+\frac{1}{2} \tilde{g}^{2}\left(1+z_{w w w w}\right) \tilde{W}_{\nu}^{+} \tilde{W}_{\sigma}^{-}-\frac{1}{2} \tilde{g}^{2} \tilde{c}_{\theta}^{2}\left(1+z_{z z w w}\right) \tilde{Z}_{\nu} \tilde{Z}_{\sigma}\right]
\end{aligned}
$$

where $S_{\mu \rho \nu \sigma}=2 g_{\mu \rho} g_{\nu \sigma}-g_{\mu \nu} g_{\rho \sigma}-g_{\mu \sigma} g_{\rho \nu}$ and

$$
z_{z w w}=\frac{c_{2 \theta}}{2 c_{\theta}^{2}}\left(\frac{g}{g^{\prime \prime}}\right)^{2} \quad z_{w w w w}=\frac{1}{4}\left(\frac{g}{g^{\prime \prime}}\right)^{2} \quad z_{z z w w}=\frac{c_{2 \theta}^{2}}{4 c_{\theta}^{4}}\left(\frac{g}{g^{\prime \prime}}\right)^{2}
$$

The electromagnetic $U(1)$ invariance is preserved.

\section{Fields and couplings renormalization}

To identify the physical quantities we define new fields in such a way to have canonical kinetic terms and to cancel the mixing term $\tilde{A}_{\mu}-\tilde{Z}_{\mu}$. They are the following:

$$
\begin{aligned}
\tilde{A}_{\mu} & =\left(1-\frac{z_{\gamma}}{2}\right) A_{\mu}+z_{z_{\gamma}} Z_{\mu} \\
\tilde{W}_{\mu}^{ \pm} & =\left(1-\frac{z_{w}}{2}\right) W_{\mu}^{ \pm} \\
\tilde{Z}_{\mu} & =\left(1-\frac{z_{z}}{2}\right) Z_{\mu}
\end{aligned}
$$

Let us study the effects of this renormalization.

First of all for the mass terms we get:

$$
-\frac{v^{2}}{4} \operatorname{tr}\left(\tilde{\mathbf{W}}_{\mu}-\tilde{\mathbf{Y}}_{\mu}\right)^{2}=-\tilde{M}_{W}^{2}\left(1-z_{w}\right) W^{\mu+} W_{\mu}^{-}-\frac{1}{2} \tilde{M}_{Z}^{2}\left(1-z_{z}\right) Z^{\mu} Z_{\mu}
$$

with

$$
\tilde{M}_{W}^{2}=\frac{v^{2}}{4} \tilde{g}^{2} \quad \tilde{M}_{Z}^{2}=\tilde{M}_{W}^{2} / \tilde{c}_{\theta}^{2}
$$

Also, the field renormalization affects all the couplings of the standard gauge bosons to the fermions. By substituting eq. (4.1) in eqs. (3.3) and (3.4) we get

$$
\begin{aligned}
\mathcal{L}_{\text {eff }}^{\text {charged }}= & -\frac{\tilde{e}}{\sqrt{2} \tilde{s}_{\theta}}\left(1-\frac{b}{2}-\frac{z_{w}}{2}\right) \bar{\psi}_{d} \gamma^{\mu} \frac{1-\gamma_{5}}{2} \psi_{u} W_{\mu}^{-}+\text {h.c. } \\
\mathcal{L}_{\text {eff }}^{\text {neutral }}= & -\frac{\tilde{e}}{\tilde{s}_{\theta} \tilde{c}_{\theta}}\left(1-\frac{b}{2}-\frac{z_{z}}{2}\right) \bar{\psi} \gamma^{\mu}\left[T_{L}^{3} \frac{1-\gamma_{5}}{2}-Q \tilde{s}_{\theta}^{2}\left(1+\frac{b}{2}-\frac{\tilde{c}_{\theta}}{\tilde{s}_{\theta}} z_{z \gamma}\right)\right] \psi Z_{\mu} \\
& -\tilde{e}\left(1-\frac{z_{\gamma}}{2}\right) \bar{\psi} \gamma^{\mu} Q \psi A_{\mu}
\end{aligned}
$$


We see that the physical constants as the electric charge, the Fermi constant and the mass of the $Z$, which are the input parameters for the physics at LEP, must be redefined in terms of the parameters appearing in our effective lagrangian. They are identified as follows

$$
\begin{aligned}
e & =\tilde{e}\left(1-\frac{z_{\gamma}}{2}\right) \\
M_{Z}^{2} & =\tilde{M}_{Z}^{2}\left(1-z_{z}\right)
\end{aligned}
$$

Concerning the Fermi constant $G_{F}$, it is evaluated from the $\mu$-decay process. Since the charged current coupling is modified by a factor $\left(1-b / 2-z_{w} / 2\right)$ and the $W$ mass

$$
M_{W}^{2}=\tilde{M}_{W}^{2}\left(1-z_{w}\right)
$$

we get

$$
\frac{G_{F}}{\sqrt{2}}=\frac{\tilde{e}^{2}\left(1-b-z_{w}\right)}{8 \tilde{s}_{\theta}^{2} \tilde{M}_{W}^{2}\left(1-z_{w}\right)}=\frac{e^{2}}{8 \tilde{s}_{\theta}^{2} \tilde{c}_{\theta}^{2} M_{Z}^{2}}\left(1-b-z_{z}+z_{\gamma}\right)
$$

where we have used eq. (4.6). We choose to define $s_{\theta}$ and $c_{\theta}$ by equating this expression to the one in the SM (tree level): $G_{F} / \sqrt{2}=e^{2} /\left(8 s_{\theta}^{2} c_{\theta}^{2} M_{Z}^{2}\right)$. We get

$$
s_{\theta}^{2} c_{\theta}^{2}=\tilde{s}_{\theta}^{2} \tilde{c}_{\theta}^{2}\left(1+b+z_{z}-z_{\gamma}\right)
$$

that is

$$
\begin{aligned}
& s_{\theta}^{2}=\tilde{s}_{\theta}^{2}\left(1+\frac{c_{\theta}^{2}}{c_{2 \theta}}\left(b+z_{z}-z_{\gamma}\right)\right) \\
& c_{\theta}^{2}=\tilde{c}_{\theta}^{2}\left(1-\frac{s_{\theta}^{2}}{c_{2 \theta}}\left(b+z_{z}-z_{\gamma}\right)\right)
\end{aligned}
$$

\section{Observables}

Let us now discuss how the effects of the $\mathbf{V}$ modifies the observables.

For the physics at LEP and TEVATRON, the modifications due to heavy particles are contained in the so-called oblique corrections. In the low-energy limit, one can expand the vacuum polarization amplitudes in $q^{2} / M_{V}^{2}$ and they can be parametrized in terms of three independent parameters. They are for example $\Delta r_{W}, \Delta k$ and $\Delta \rho$ or, equivalently, the $\epsilon$ parameters [10].

Let us start from $\Delta r_{W}$. It is customary define

$$
\frac{M_{W}^{2}}{M_{Z}^{2}}=c_{\theta}^{2}\left[1-\frac{s_{\theta}^{2}}{c_{2 \theta}} \Delta r_{W}\right]
$$

From the relation $\tilde{M}_{W}^{2}=\tilde{M}_{Z}^{2} \tilde{c}_{\theta}^{2}$ we get

$$
\frac{M_{W}^{2}}{M_{Z}^{2}}=c_{\theta}^{2}\left[1+z_{z}-z_{w}-\frac{s_{\theta}^{2}}{c_{2 \theta}}\left(-b+z_{\gamma}-z_{z}\right)\right]
$$

so, for comparison, and using eq. (3.7)

$$
\Delta r_{W}=-b+z_{\gamma}+\frac{c_{2 \theta}}{s_{\theta}^{2}} z_{w}-\frac{c_{\theta}^{2}}{s_{\theta}^{2}} z_{z}=-b+2\left(\frac{g}{g^{\prime \prime}}\right)^{2}
$$


The definitions of $\Delta \rho$ and $\Delta k$ are given in terms of the neutral current coupling to the $Z$

$$
\mathcal{L}^{\text {neutral }}(Z)=-\frac{e}{s_{\theta} c_{\theta}}\left(1+\frac{\Delta \rho}{2}\right) Z_{\mu} \bar{\psi}\left[\gamma^{\mu} g_{V}+\gamma^{\mu} \gamma_{5} g_{A}\right] \psi
$$

with

$$
\begin{aligned}
g_{V} & =\frac{T_{L}^{3}}{2}-s_{\bar{\theta}}^{2} Q \\
g_{A} & =-\frac{T_{L}^{3}}{2} \\
s_{\bar{\theta}}^{2} & =(1+\Delta k) s_{\theta}^{2}
\end{aligned}
$$

By using eqs. (4.6) and (4.10) we get

$$
\frac{e}{s_{\theta} c_{\theta}}=\frac{\tilde{e}}{\tilde{s}_{\theta} \tilde{c}_{\theta}}\left(1-\frac{b}{2}-\frac{z_{z}}{2}\right)
$$

For comparison with eq. (4.5), and using eq. (3.7), we obtain

$$
\begin{aligned}
\Delta \rho & =0 \\
\Delta k & =\frac{c_{\theta}^{2}}{c_{2 \theta}}\left(z_{\gamma}-z_{z}\right)-\frac{c_{\theta}}{s_{\theta}} z_{z \gamma}-\frac{1}{2 c_{2 \theta}} b=\frac{1}{c_{2 \theta}}\left[-\frac{b}{2}+\left(\frac{g}{g^{\prime \prime}}\right)^{2}\right]
\end{aligned}
$$

Summarizing, we have the following correspondence between corrections and observables: $\Delta r_{W}$ is equivalent to $M_{W} / M_{Z}$ which is measured at TEVATRON, $\Delta k$ modifies the vector coupling $g_{V}$ and $\Delta \rho$ modifies the neutral coupling overall strength. At LEP, $\Delta k$ can be obtained by measuring the forward-backward asymmetry at the $Z$ peak. Then, having fixed $\Delta k, \Delta \rho$ can be determined by the leptonic width. All these quantities receive contributions also from weak radiative corrections. In particular they depend quadratically from the top mass which is still affected by a large error. From the point of view of data analysis it turns out to be more convenient to isolate such contribution in $\Delta \rho$ and define two other linear combinations which depend only logarithmically on $m_{\text {top }}$. They are the so-called $\epsilon$ parameters [10]

$$
\begin{aligned}
\epsilon_{1} & =\Delta \rho \\
\epsilon_{2} & =c_{\theta}^{2} \Delta \rho+\frac{s_{\theta}^{2}}{c_{2 \theta}} \Delta r_{W}-2 s_{\theta}^{2} \Delta k \\
\epsilon_{3} & =c_{\theta}^{2} \Delta \rho+c_{2 \theta} \Delta k
\end{aligned}
$$

Using eqs. (5.3) and (5.7) we get

$$
\begin{aligned}
\epsilon_{1} & =0 \\
\epsilon_{2} & =0 \\
\epsilon_{3} & =-\frac{b}{2}+\left(\frac{g}{g^{\prime \prime}}\right)^{2}
\end{aligned}
$$

This extends the previous results obtained in $[6]$ to the case $b \neq 0$.

We can derive restrictions on the BESS parameters by using the experimental data on $\epsilon_{3}$. The most recent value for $\epsilon_{3}$ obtained by combining the LEP, low-energies, CDF/UA2 and SLD data [1] gives

$$
\epsilon_{3}=(3.9 \pm 1.7) \times 10^{-3}
$$


By assuming for the BESS model the same one-loop radiative corrections as for the SM in which the Higgs mass is used as a cut-off $\Lambda$ [1], we add to $\epsilon_{3}$ given in eq. (5.9) the contribution coming from the radiative corrections [11] calculated for $M_{H}=\Lambda=1 \mathrm{TeV}$ and $m_{\text {top }}=(174 \pm 17) \mathrm{GeV}$, which are $\left(\epsilon_{3}\right)_{\text {rad.corr } .} \simeq\left(6.39_{+0.20}^{-0.14}\right) \times 10^{-3}$. The allowed region at $90 \%$ C.L. in the plane $\left(b, g / g^{\prime \prime}\right)$ is shown in Fig. 1. By increasing the value of the top mass, the region moves slightly to the left (the solid (dashed) line is for $m_{t}=$ 191(157) $\mathrm{GeV})$.

For completeness, we derive the correction to the charged current coupling which is defined as follows

$$
\mathcal{L}^{\text {charged }}=-h_{W} \bar{\psi}_{d} \gamma^{\mu} \frac{1-\gamma_{5}}{2} \psi_{u} W_{\mu}^{-}+\text {h.c. }
$$

where, in the SM, $h_{W}=e /\left(\sqrt{2} s_{\theta}\right)$. By comparing with eq. (4.4) and using eqs. (4.6) and (4.10) we get

$$
h_{W}=\frac{e}{\sqrt{2} s_{\theta}}\left[1+\frac{z_{\gamma}}{2}-\frac{z_{w}}{2}-\frac{b}{2}+\frac{c_{\theta}^{2}}{2 c_{2 \theta}}\left(z_{z}-z_{\gamma}+b\right)\right]=\frac{e}{\sqrt{2} s_{\theta}}\left[1-\frac{s_{\theta}^{2}}{c_{2 \theta}} \epsilon_{3}\right]
$$

where we have used the eqs. (3.7) and (5.9).

It is worth to stress that, in the low-energy regime (that is up to the mass of the $Z$ ), all deviations to the Standard Model due to BESS are contained in $\epsilon_{3}$, which turns out to be the sum of two contributions, one from the direct coupling of $\mathbf{V}$ to fermions, and the other coming out from the mixing of $\mathbf{V}$ with the ordinary gauge bosons. As a consequence the low energy experiments restrict the plane $\left(b, g / g^{\prime \prime}\right)$ only to a strip (see Fig. 1), and not to a closed region. However, more informations can be obtained by studying the trilinear couplings.

\section{Anomalous trilinear and quadrilinear gauge cou- plings}

The corrections to the trilinear and quadrilinear gauge couplings come from three different sources: from the $\mathbf{V}$ kinetic term, after the substitution of the equation of motion for the $\mathbf{V}$ fields, from the $\mathbf{W}$ and $\mathbf{Y}$ kinetic terms after renormalization, from the renormalization of the couplings.

Let us perform the renormalization of the fields and couplings as defined in Sect. 4 to evaluate the anomalous contributions to the trilinear and quadrilinear gauge couplings given in eqs. (3.8) and (3.9). The result is the following

$$
\begin{aligned}
& \mathcal{L}_{e f f}^{\text {kin }}{ }^{(3)}\left(W^{ \pm}, A, Z\right)=i g c_{\theta}(1+k)\left[Z^{\mu \nu} W_{\mu}^{-} W_{\nu}^{+}+Z^{\nu}\left(W_{\mu \nu}^{-} W_{\mu}^{+}-W_{\mu \nu}^{+} W_{\mu}^{-}\right)\right] \\
& +i e\left[A^{\mu \nu} W_{\mu}^{-} W_{\nu}^{+}+A^{\nu}\left(W_{\mu \nu}^{-} W_{\mu}^{+}-W_{\mu \nu}^{+} W_{\mu}^{-}\right)\right] \\
& \mathcal{L}_{\text {eff }}^{k i n}{ }^{(4)}\left(W^{ \pm}, A, Z\right)=S_{\mu \rho \nu \sigma} W_{\mu}^{+} W_{\rho}^{-}\left[-\frac{e^{2}}{2} A_{\nu} A_{\sigma}-e g c_{\theta}(1+k) A_{\nu} Z_{\sigma}\right. \\
& \left.+\frac{1}{2} g^{2}\left(1+k_{1}\right) W_{\nu}^{+} W_{\sigma}^{-}-\frac{1}{2} g^{2} c_{\theta}^{2}\left(1+k_{2}\right) Z_{\nu} Z_{\sigma}\right]
\end{aligned}
$$


with

$$
\begin{aligned}
k & =\frac{1}{2 c_{2 \theta}} b-\frac{1}{2 c_{\theta}^{2} c_{2 \theta}}\left(\frac{g}{g^{\prime \prime}}\right)^{2} \\
k_{1} & =\frac{c_{\theta}^{2}}{c_{2 \theta}} b+\left(\frac{1}{4}-\frac{1}{c_{2 \theta}}\right)\left(\frac{g}{g^{\prime \prime}}\right)^{2} \\
k_{2} & =\frac{1}{c_{2 \theta}} b-\frac{1+2 c_{\theta}^{2}}{4 c_{\theta}^{4} c_{2 \theta}}\left(\frac{g}{g^{\prime \prime}}\right)^{2}
\end{aligned}
$$

If we want to write an effective Lagrangian containing terms up to the order $p^{4}$ (the gauge fields are formally considered of the order $p$ ) then we have other invariant terms which can contribute to the anomalous trilinear and quadrilinear couplings among the SM gauge bosons. For example, two invariant terms which preserve $C P$ and $L \leftrightarrow R$ invariances, are the following (see the second paper in ref. [3])

$$
\begin{aligned}
L_{I} & =\gamma \operatorname{tr}\left(F^{\mu \nu}(\mathbf{V})\left[\omega_{\mu}^{\perp}, \omega_{\nu}^{\perp}\right]\right) \\
L_{I I} & =\delta \operatorname{tr}\left(F^{\mu \nu}(\mathbf{V})\left[\omega_{\mu}^{\|}-\mathbf{V}_{\mu}, \omega_{\nu}^{\|}-\mathbf{V}_{\nu}\right]\right)
\end{aligned}
$$

Let us substitute the expressions (2.3). Then, after the $S U(2)_{L} \otimes U(1)_{Y}$ gauging through eq. (2.8), we get, in the unitary gauge

$$
\begin{aligned}
L_{I} & =\frac{\gamma}{4} \operatorname{tr}\left(F^{\mu \nu}(\mathbf{V})\left[\tilde{\mathbf{W}}_{\mu}-\tilde{\mathbf{Y}}_{\mu}, \tilde{\mathbf{W}}_{\nu}-\tilde{\mathbf{Y}}_{\nu}\right]\right) \\
L_{I I} & =\frac{\delta}{4} \operatorname{tr}\left(F^{\mu \nu}(\mathbf{V})\left[\tilde{\mathbf{W}}_{\mu}+\tilde{\mathbf{Y}}_{\mu}-2 \mathbf{V}_{\mu}, \tilde{\mathbf{W}}_{\nu}+\tilde{\mathbf{Y}}_{\nu}-2 \mathbf{V}_{\nu}\right]\right)
\end{aligned}
$$

Notice that if we add these two terms to the BESS Lagrangian (2.11), the solution of the classical equations of motion for $\mathbf{V}$ are again given by eq. (3.1), because the contributions from $L_{I}$ and $L_{I I}$ vanish in the limit $\alpha \rightarrow \infty$. Furthermore, when substituting the classical solution (3.1), we see that only $L_{I}$ survives in the effective Lagrangian. By performing the explicit calculations and separating the trilinear from the quadrilinear terms, we get

$$
\begin{gathered}
\left(L_{I}\right)_{e f f}^{(3)}\left(W^{ \pm}, A, Z\right)=\quad i \gamma \frac{g^{2}}{4}\left[g \frac{c_{2 \theta}}{2 c_{\theta}} Z^{\mu \nu} W_{\mu}^{-} W_{\nu}^{+}+\frac{g}{2 c_{\theta}} Z^{\nu}\left(W_{\mu \nu}^{-} W_{\mu}^{+}-W_{\mu \nu}^{+} W_{\mu}^{-}\right)\right. \\
\left.+e A^{\mu \nu} W_{\mu}^{-} W_{\nu}^{+}\right] \\
\left(L_{I}\right)_{e f f}^{(4)}\left(W^{ \pm}, A, Z\right)= \\
\quad \frac{g^{2}}{4} S_{\mu \rho \nu \sigma} W_{\mu}^{+} W_{\rho}^{-}\left[-e \frac{g}{2 c_{\theta}} A_{\nu} Z_{\sigma}\right. \\
\left.-\frac{g^{2}}{4} W_{\nu}^{+} W_{\sigma}^{-}-g^{2} \frac{c_{2 \theta}}{4 c_{\theta}^{2}} Z_{\nu} Z_{\sigma}\right]
\end{gathered}
$$

Notice that since we want to recover the SM Lagrangian in the $g^{\prime \prime} \rightarrow \infty$ limit, we consider $\gamma$ of the order of $1 / g^{\prime \prime}$. In this way, it is not necessary to perform the renormalization of the fields and couplings as described in Sect. 4 since all those corrections give contributions at least of the order $\left(1 / g^{\prime \prime}\right)^{3}$ which are negligible in our context. 
Concerning the anomalous trilinear terms, we end up with the following observation. By comparing with ref. [12 we can extract relations among our parameters $k$ and $\gamma$ and the definitions used in the literature to parametrize the deviations from the SM trilinear couplings to be tested at the future colliders. We get

$$
x_{\gamma}=\gamma \frac{g^{2}}{4} \quad \delta_{z}=\frac{c_{\theta}}{s_{\theta}}\left(k+\gamma \frac{g^{2}}{8 c_{\theta}^{2}}\right) \quad x_{z}=-\gamma \frac{g^{2}}{4} \frac{s_{\theta}}{c_{\theta}}
$$

where $\delta_{z}$ describes a deviation of the $Z W^{+} W^{-}$overall coupling from the standard value while $x_{\gamma}$ and $x_{z}$ parametrize the potential deviations in the electromagnetic and weak dipole couplings from the SM predictions.

We see that, for $\gamma=0$, there is only one parameter different from zero, $\delta_{z}$, whereas for $k=0$ (that is for $b=\left(g / g^{\prime \prime}\right)^{2}\left(1 / c_{\theta}^{2}\right)$ see eq. (6.3)) we have again only one free parameter, say $x_{\gamma}$, and the following relations hold

$$
\begin{aligned}
\delta_{z} & =\frac{x_{\gamma}}{2 s_{\theta} c_{\theta}} \\
x_{z} & =-\frac{s_{\theta}}{c_{\theta}} x_{\gamma}
\end{aligned}
$$

So, in the general case with $k$ and $\gamma$ different from zero, BESS is a combination of the previous situations, and eq. (6.10) always holds.

\section{Discussion}

The analysis presented here shows that low energy experiments do not put really stringent bounds in the plane $\left(b, g / g^{\prime \prime}\right)$ of the BESS parameter space. However, when there will be the possibility to add to the analysis the experiments aimed to measure the anomalous couplings (as in the $e^{+} e^{-}$colliders), we will be able to fix the set of parameters $\left(b, g / g^{\prime \prime}, \gamma\right)$, in terms of $\epsilon_{3}$, and of the anomalous couplings $x_{\gamma}, \delta_{z}$ and $x_{z}$. In this situation the relation (6.10) would represent a test of the model. Unfortunately it is impossible to use in the present context the quantitative bounds for the anomalous couplings given in the literature (see, for instance ref. [12]). In fact it is generally assumed that $Z$ and $W$ have standard couplings to the fermions, which is not the case here, because the effective couplings are modified by terms proportional to $\epsilon_{3}$. A precise analysis must take into account this effect in a proper way. However if we assume that there are no cancellations among the various contributions we can still make a qualitative analysis. First of all we recall that the parameter $\gamma$ is naturally expected to be of order $1 / g^{\prime \prime}$, because we want that the $V$ bosons decouple in the limit $g^{\prime \prime} \rightarrow \infty$. Then let us consider two possibilities.

i) In $\epsilon_{3}$ there is no accidental cancellation between the $b$ term and $\left(g / g^{\prime \prime}\right)^{2}$. In this case both quantities should be of order $\left(\epsilon_{3}\right)_{\text {exp. }}-\left(\epsilon_{3}\right)_{\text {rad.corr. }} \approx 10^{-3}$ and the same would be for $k$ defined in eq. (6.3). As far as $\gamma$ is concerned we can write $\gamma=\tilde{\gamma} / g^{\prime \prime}$ and assume $\tilde{\gamma} \approx \mathcal{O}(1)$. From the previous estimate we get $\left(g / g^{\prime \prime}\right) \approx 3 \%$ and therefore $\gamma g^{2} / 4 \approx 5 \times 10^{-3}$. In this case we get both $\delta_{z}$ and $x_{\gamma} \approx 5 \times 10^{-3}$. This means that, by considering the results of 112 for the case of a two parameters model, even a $1 \mathrm{TeV} e^{+} e^{-}$collider is not enough to test these trilinear anomalous couplings.

ii) There is an accidental cancellation in $\epsilon_{3}, b / 2=\left(g / g^{\prime \prime}\right)^{2}$. In this case

$$
k=\frac{1}{2 c_{\theta}^{2}}\left(\frac{g}{g^{\prime \prime}}\right)^{2}
$$


This time there are no low-energy restrictions on $g^{\prime \prime}$. However we are working in the limit $g^{\prime \prime}>>g$, which in practice means that $g / g^{\prime \prime}$ should be something less than, say, $10 \%$, and the contribution of this term to $\delta_{z}$ would be less that $1 \%$. Analogously the $\gamma$-contribution is of the same order giving $x_{\gamma} \approx 1.5 \%$. From ref. [12] one can see that an $e^{+} e^{-}$collider somewhat in between $500 \mathrm{GeV}$ and $1 \mathrm{TeV}$ would be enough for testing these couplings.

A much more complete analysis of the restrictions of the BESS parameters on the plane $\left(b, g / g^{\prime \prime}\right)$, for $\gamma=0$, can be found in ref. [13]. 


\section{References}

[1] S.Weinberg, Phys. Rev. D13 (1976) 974; D19 (1979) 1277; L.Susskind, Phys. Rev. D20 (1979) 2619; for a review see E.Farhi and L.Susskind, Phys. Rep. 74 (1981) 277

[2] M.Chanowitz and M.K.Gaillard, Nucl. Phys. B261 (1985) 379, and references therein

[3] R.Casalbuoni, S.De Curtis, D.Dominici and R.Gatto, Phys. Lett. B155 (1985) 95; Nucl. Phys. B282 (1987) 235

[4] R.Casalbuoni, S.De Curtis, D.Dominici, F. Feruglio and R.Gatto, Phys. Lett. B269 (1991) 361

[5] A.Dobado, M.J.Herrero and J.Terron, Proceedings of "Large Hadron Collider Workshop", Vol. II, pag. 770, Aachen, 4-9 October, 1990

[6] R.Casalbuoni, S.De Curtis, D.Dominici, F.Feruglio and R.Gatto, Phys. Lett. B258 (1991) 161

[7] R.Casalbuoni, S.De Curtis, D.Dominici F.Feruglio and R.Gatto, Int. Jour. Mod. Phys. A4 (1989) 1065; R.Casalbuoni, A. Deandrea, S.De Curtis, N.Di Bartolomeo, D.Dominici, F.Feruglio and R.Gatto, Nucl. Phys. B409 (1993) 257

[8] C.P.Burges et al., Phys. Rev. D49 (1994) 6115

[9] A.P.Balachandran, A.Stern and G.Trahern, Phys. Rev. D19 (1979) 2416; M.Bando, T.Kugo and K.Yamawaki, Phys. Rep. 164 (1988) 217

[10] G.Altarelli, R.Barbieri and F.Caravaglios, Nucl.Phys. B405 (1993) 3, and references therein

[11] G.Altarelli, CERN Preprint, CERN-TH 7464/94 (1994)

[12] M.Bilenky, D.Schildnecht, J.-L.Kneur and F.M.Renard, DESY93-123C, p. 187 (1993)

[13] R.Casalbuoni, P.Chiappetta, A. Deandrea, S.De Curtis, D.Dominici, F.Feruglio and R.Gatto, Z. für Physik C60 (1993) 315

\section{Figure Captions}

Fig. 1 Allowed region at $90 \%$ C.L. in the plane $\left(b, g / g^{\prime \prime}\right)$ for the BESS model coming from the measurement of $\epsilon_{3}$. The solid (dashed) line is for $m_{t}=191$ (157) $\mathrm{GeV}$ and $\Lambda=1 \mathrm{TeV}$. 
This figure "fig1-1.png" is available in "png" format from: http://arxiv.org/ps/hep-ph/9410377v1 


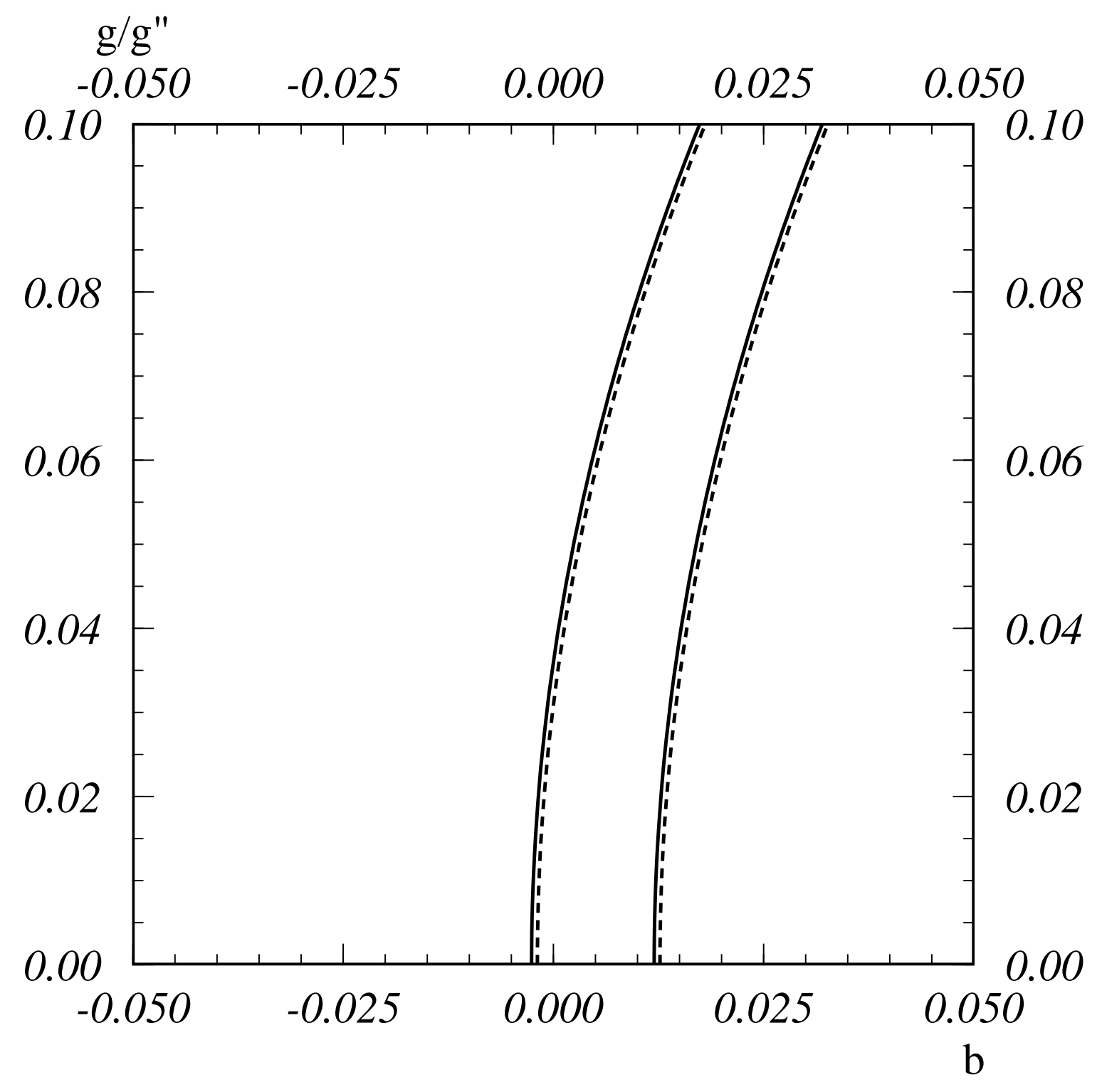

Fig. 1 